\title{
Determination of Wood Moisture Properties Using a CT-Scanner in a Controlled High-Temperature Environment
}

\author{
Lars Hansson and Ekaterina Cherepanova \\ Department of Engineering Sciences and Mathematics, Division of Wood Science and Engineering, Wood Physics, Skellefteå, Sweden
}

\begin{abstract}
Theaim of the present work wasto analyze moisture flow and moisture content data for high-temperature drying by usingan advanced image- processing algorithm.Since wood starts to shrink below the fibre saturation point during drying, the size and shape of wood will change. The dry wood image was thoroughly transformed to the shape of the wet wood image prior to calculating the dry weight moisture content. The use of the image- processing algorithm for the dry weight moisture content on density data from the CT-scanning during drying in a controlled high-temperature environment showed that this method is a powerful tool for analyzing the moisture flow inside the wood piece. Furthermore, the new CT-scanner together with the climate chamber gave unique results, as it has not been possible to study high-temperature drying with this method before.
\end{abstract}

Key words: CT-scanning, fibre saturation point, high-temperature drying, image analysis, moisture content, wood drying.

\section{Introduction}

Wood drying is a complex physical process, during which heat and mass transfer are coupled. One of the most common drying methods nowadays is forced air-circulation. This drying is based on heat conduction from the surface of the wood towards the interior for evaporation of moisture. The moisture moves to the surface by mass flow in liquid and vapour phases. Water can exist in several states in the wood structure: as free water, as bound water in the wood cell wall and as water vapour in the voids. Regardless of which kind of air-circulation drying is used, high or low temperature, the water flow within the wood depends on several different physical mechanisms.

Hawley [1] has explained these mechanisms during drying. When drying wood it is possible to clearly see the higher level of water in the narrower capillaries, the general decrease of the mean water level as drying proceeds and the presence of air bubbles in individual

Corresponding author: Lars Hansson, associate professor, research fields: wood physics. E-mail: lars.hansson@ltu.se. cells still filled with water, which expand and gradually fill the entire cell cavity [1]. Further drying of the wood below the fibre saturation point(fsp) results in strengthening of the wood fibre sand is usually accompanied by shrinkage.

Many researchers have developed physical models of the movement of water through wood during high-temperature (HT) drying. For HT drying, simulation models must take into account the vapour partial pressure gradient as a driving force for the moisture content $(\mathrm{mc})$ movement during drying [2]. With this assumption, Pang [2] has shown that the drying models give predictions that are in agreement with observed data. By adding a pressure equation to an existing drying model of heat and mass transfer, Nabhani [3] showed a good agreement between predicted curves and the evolution of gas pressure in the board centre. Nijdam [4] has shown, by comparison between experiments, the importance of accounting for the orientation of the growth rings to the drying surface and the effect of density variations on the liquid 
permeability, especially at high core dry weight mc in the HT drying model.

By using an X-ray computed tomography CT-scanner, Wiberg [5] has analysed moisture distribution and moisture flux above the fsp for different kinds of wood during low-temperature drying. With this method advanced non-destructive studies could be done of the internal characteristics of wood. The CT-scanner which was used [5] has been replaced. Besides, a new purpose-built climate chamber has been installed as a complement to the new CT-scanner, as the maximum temperature that can be reached in this climate chamber is $215^{\circ} \mathrm{C}$ compared to the earlier $80^{\circ} \mathrm{C}$.

In timber production, wood drying is one of the most important parts. It takes the longest time in the processing chain and uses most of the energy. By using the new purpose-built climate chamber with the CT-scanner during HT drying, physical wood properties could be investigated and the result could be used for predictive models and for developing HT-efficient drying schedules.

The aim of the present work was to use an image processing algorithm for mc calculation and to analyse the moisture flow data for HT drying.

\section{Materials and Methods}

\subsection{Principles of a CT-scanner and the Climate Chamber}

The climate chamber is designed in order to work with the CT-scanner (Fig. 1).

In simple terms, the climate chamber is designed with an inner and an outer tube, where the air, driven by a fan, flows through the heating coils and then into the inner tube, where the material is placed for the drying or treatment (Fig. 2).

At the end of the inner tube, the air flow returns to the fan by flowing in the outer tube. To increase the humidity in the chamber a steam generator is used. The climate chamber can also carry out conditioning by water spray and steam in order to equalize the moisture gradient, which may be caused by intense drying. The highest temperature that can be achieved in the kiln is $215^{\circ} \mathrm{C}$. High- and low-temperature drying processes can be carried out in the kiln as well as heat treatment processes of wood.

The control schedule, which has been developed by Valutec A. B. [7], is built up by assigning different control parameters in different phases.

\subsection{The Algorithm for Moisture Content Calculation}

Since wood starts to shrink below the fsp, the size and shape of the wood specimen will change (Fig. 3).

This means that the dry wood image must be thoroughly transformed to the shape of the wet wood image prior to calculating the $\mathrm{mc}$ [8]. By using bidirectional elastic registration (bUnwarpJ) [9], the dry wood image can be transformed to the shape of the wet wood image (Fig. 3). This algorithm (bUnwarpJ) for elastic and consistent image registration is developed as a plug-in to the software Image $\mathrm{J}$ (Image processing and analysis in Java) [10]. It is performed as a simultaneous registration of two images, in this case the dry and wet wood images. The dry wood image is elastically deformed to make it look similar as the wet wood image and, at the same time, the "inverse" transformation (from wet wood to dry wood) is also calculated so a pseudo-invertibility of the final deformation can be guaranteed. Two images are produced as a result; the deformed versions of dry and wet wood images.

By using the same approach as Hansson [8], the transformation of the CT-images and the mc calculation was done as follows:

When the mc exceeds the fsp, the green volume is determined by Eq. (1).

$$
V_{\text {green }}=\frac{V_{o}}{\left(1-\beta_{\max }\right)}
$$

where $V_{o}$ is the dry volume of wood and $\beta_{\max }$ is the maximum shrinkage coefficient [11].

It is necessary to distinguish between wet and dry volume, mass and density expressions. Combining the 

a Controlled High-Temperature Environment

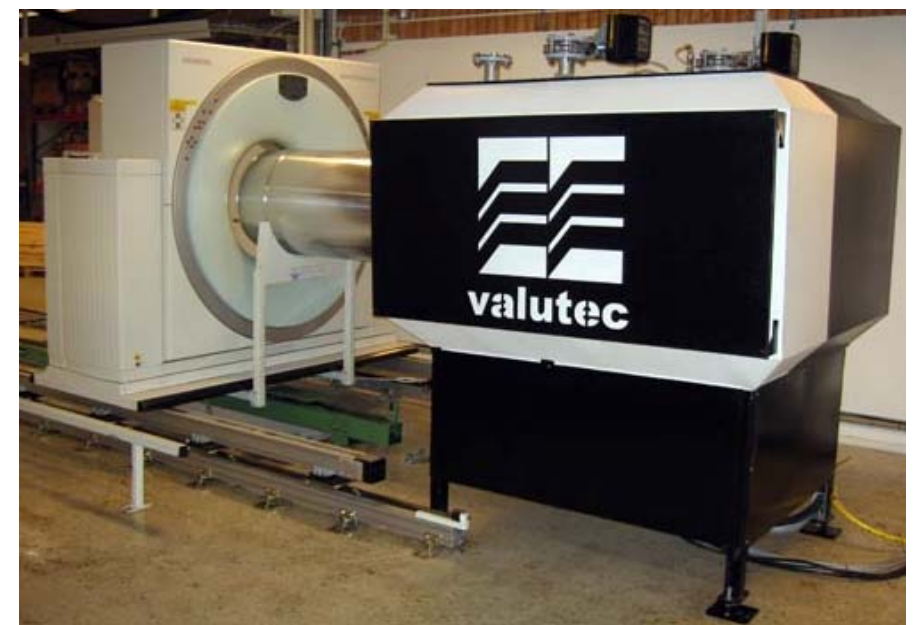

Fig. 1 CT-scanner together with the climate chamber.

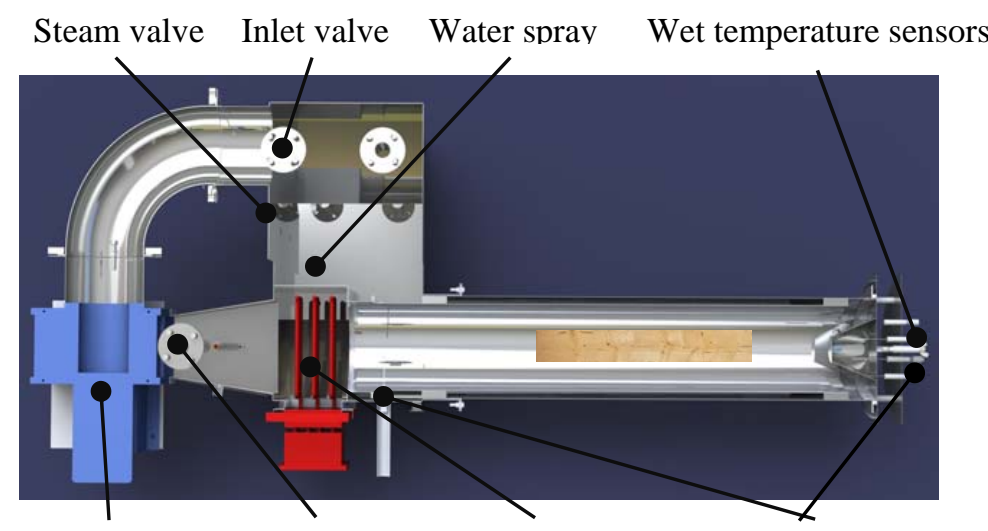

Circulation Outlet valve Heating coils Dry temperature sensors

Fig. 2 Exploded drawing of the climate chamber.
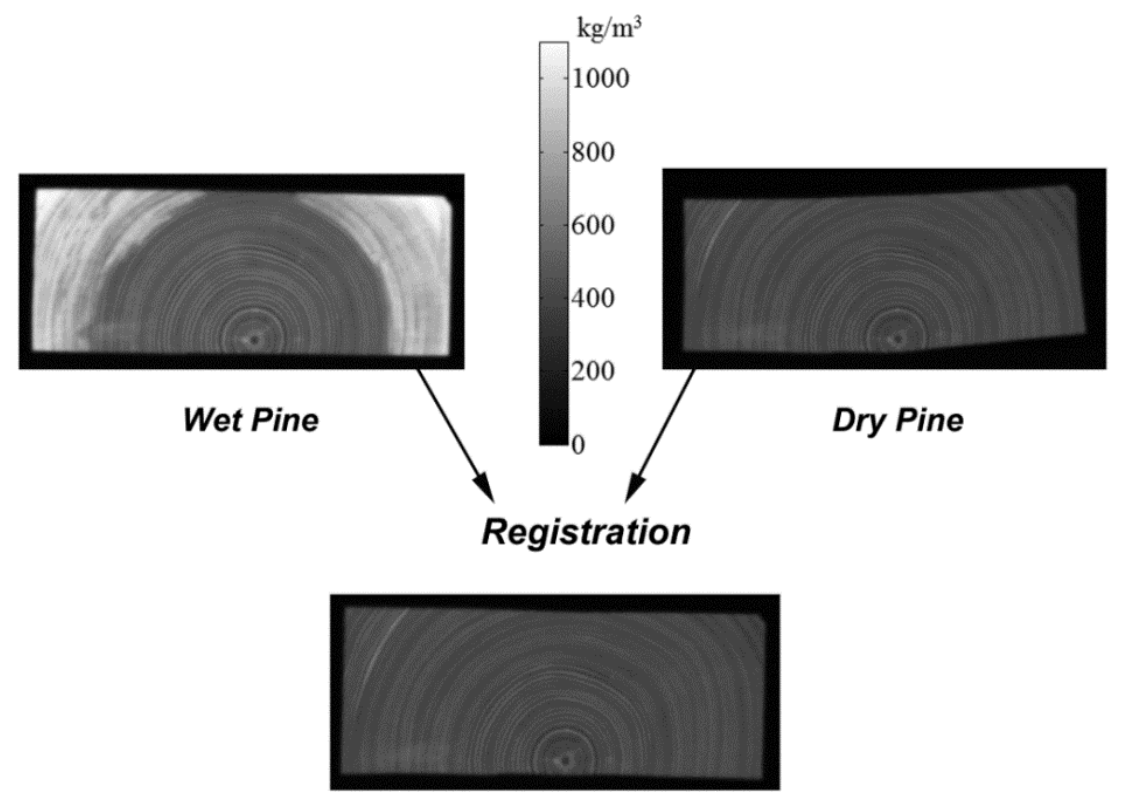

Dry piece of Pine after elastic registration

Fig. 3 Elastic registration of a pine specimen. 
definitions of mc and density of wet wood $\left(\rho_{x, x}\right)$ and dry wood $\left(\rho_{0,0}\right)$, Eq. (1) gives an expression for mc based on wet and dry density values:

$$
X_{m}=\frac{\rho_{x, x}-\rho_{o, o}\left(1-\beta_{\max }\right)}{\rho_{0,0}\left(1-\beta_{\max }\right)}, X_{m} \geq X_{f s p}
$$

However, if the mc is below the fsp, the total wet volume will be written as:

$$
V_{x}=V_{\text {green }}\left(1-\beta_{x}\right)=\frac{V_{o}}{\left(1-\beta_{\max }\right)}\left(1-\beta_{x}\right)
$$

Where $\beta_{x}$ is the shrinkage coefficient at mc below the fsp and $V_{x}$ is the total volume at mc below the fsp:

$$
\beta_{x}=\frac{\beta_{\max }}{X_{f s p}}\left(X_{f s p}-X_{m}\right)
$$

Where $X_{f s p}$ is the mc at the fsp, which is dependent on species and temperature [12]. However, it has a maximal value of approximately 0.3 at $20^{\circ} \mathrm{C}$. If the temperature increases, the fsp [13] will decrease:

$$
X_{f s p}=0.30-0.001(T-20)
$$

Where $T$ is the temperature in Celsius.

The corresponding expression for mc below the fsp, combining the definitions of mc, density of wet wood and dry wood and Eqs. (3) and (4), is:

$$
X_{m}=\frac{\left(1-\beta_{\max }\right) X_{f s p}\left(\rho_{x, x}-\rho_{0,0}\right)}{\left(\rho_{0,0}\left(1-\beta_{\max }\right) X_{f s p}-\rho_{x, x} \beta_{\max }\right)} X_{m}<X_{f s p}
$$

The algorithm for the mc calculation in the model works as follows: If the result of Eq. (6) is higher than the mc at the fsp, the mc is recalculated by Eq. (2).

The algorithm for the mc calculation in the model works as follows: If the result of Eq. (6) is higher than the mc at the fsp, the mc is recalculated by Eq. (2).

The calculation of mc sometimes gives a very small amount of unrealistic values, for which reason the calculation results need to be filtered. These unrealistic values arise in the process of transformation of the CT-scanned images. The images deviate in position relative to each other, in most cases at the wood area border. The filtering is done when an unreal value, such as negative $\mathrm{mc}$, is found by replacing it with an average of the surrounding values.

\subsection{Material and Drying Temperatures}

For the HT experiment, sapwood pieces with dimensions of $35 \times 70 \times 400 \mathrm{~mm}$ and $24 \times 66 \times 400 \mathrm{~mm}$ were freshly cut out from logs of pine. The ends were sealed with one layer of silicon closest to the wood surface and then one layer of aluminum foil, followed by an additional layer of silicon. During the drying process, the temperature of the wood pieces was measured by thermocouples in two positions: in the core of the board and $5 \mathrm{~mm}$ below the surface. The thermocouples were previously calibrated installed into drilled holes and sealed with silicon. For the HT experiment, the pieces were dried at $90 / 110^{\circ} \mathrm{C}$ (wet bulb/dry bulb temperature). To reach the dry density, the pieces were dried at $103^{\circ} \mathrm{C}$ for 24 hours.

\subsection{Scan plan and Calculation Description}

Ten centimeters of the middle part of each board were CT-scannedwith a $5 \mathrm{~mm}$ slice width every 15 minutes. Thus 20 density images were obtained for each time period. With the previously described algorithm, the mc distribution was calculated for each time step. Furthermore, the average mc and density for a selected core region were determined for every time step. The selected core region had dimensions of $130 \times 20$ pixels $(75.4 \times 11.6 \mathrm{~mm})$ (Fig. 4).

Hence, the rate of moisture flow from the core versus the average mc could be calculated. The moisture flow is calculated by taking the difference between the densities for each time step.

\section{Results and Discussion}

By using the method for calculating the dry weight $\mathrm{mc}$, the moisture loss from the core can be calculated (Fig. 5, left).

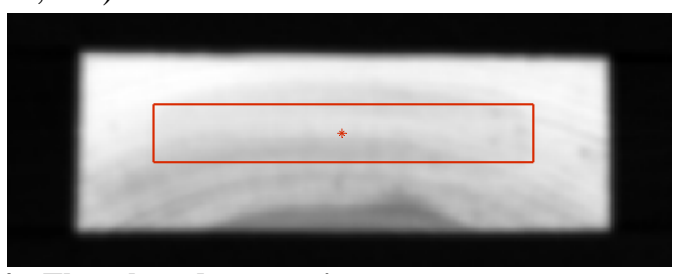

Fig. 4 The selected core region. 

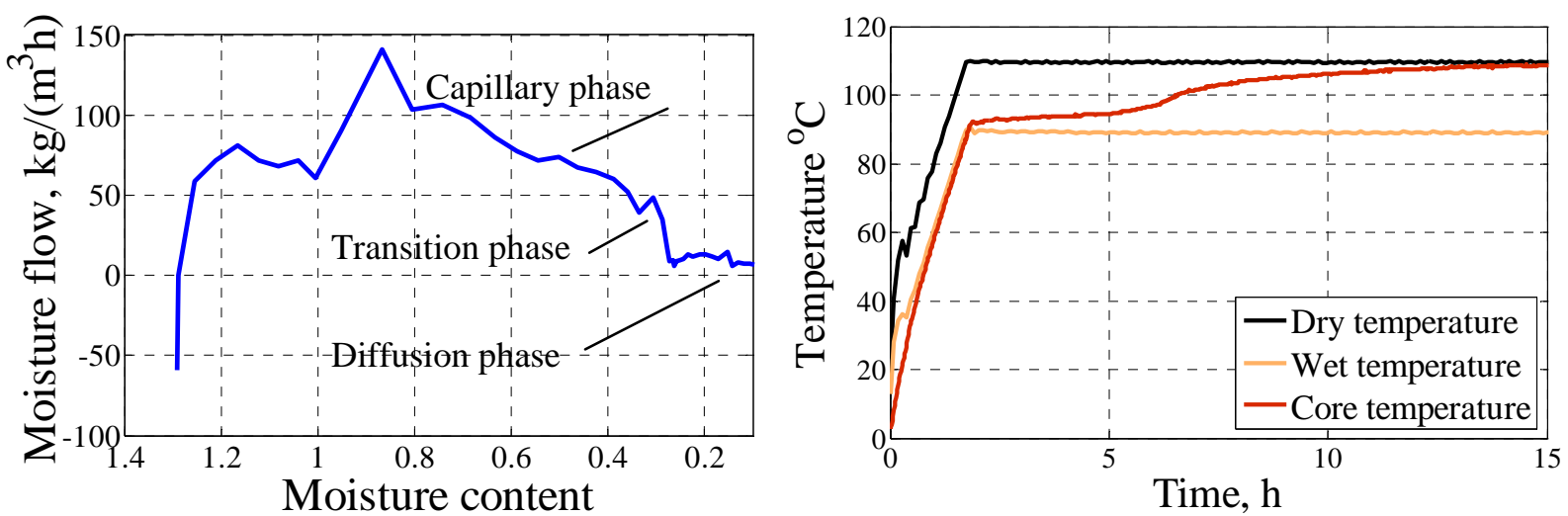

Fig. 5 The rate of moisture loss from the core vs. the average dry weight moisture content (left) and the core temperature vs. time (right).

The rise in the temperature in the kiln makes a big difference between the wood core and the dry bulb temperature inside the kiln (Fig. 5, right).

As the temperature increases, the density of wood decreases and this creates a pressure difference. Since the temperature is proportional to pressure, the water and vapour pressure is much higher in the region close to the surface than in the wood core. The temperature difference makes the moisture lossto the internal part of the wood piece. This phenomenon is called Darcy flow, which explains the negative moisture loss at the beginning of drying (Fig. 5, left) as well as the density increasing moisture flow (Fig. 6). After about half an hour, the wet bulb temperature in the kiln becomes equal to the wood temperature. Furthermore, the water starts to move towards the surface from the core as it begins to evaporate from the surface.

The rising core temperature after around half an hour leads to an accelerated moisture loss from the core. The evaporation of water from the surface sets up capillary forces that exert a pull on the free water in the zones of wood beneath the surfaces. The drying process is now in the capillary phase and this phase ends after approximately 4.5 hours. When there is no longer any free water in the wood, capillary forces are no longer of importance. By studying the moisture loss in the density vs. time plot (Fig. 6), one can see that it decreases linearly in the defined core area from about 0.5 hours to 4.5 hours, which means that the moisture profiles decrease almost uniformly as long as there is capillary communication (Fig. 6). The average moisture loss versus dry mc (Fig. 5, left) shows that the moisture loss decreases constantly from 0.8 to around 0.3 . Suddenly the core temperature starts to rise after 5 hours (Fig. 5, right), which means that the drying process is going into the transition phase and the rate of moisture loss from the core versus the average dry weight mc will drop dramatically (Fig. 5, left). Furthermore, the average density change starts to decrease (Fig. 6). The moisture profile (Fig. 6) shows mc "islands", which also indicates that the drying process is in the transition phase. Hence, the capillary connection has begun to break down.

After the transition phase, with a dry weight mc of about 0.28 , the core temperature increases faster (Fig. 5 , right) and the rate of moisture loss from the core versus the average dry weight moisture (Fig. 5, left) is almost constant. Furthermore, the moisture flow versus time decreases only slowly at present. The drying process is now in the diffusion phase and moisture slowly evaporates from the wood piece.

The distribution of mc inside the wood piece is irregular during drying (Fig. 7). By comparing the cross-section's image for the mc and dry density, it can be seen that the high density (brighter) part dries faster than the low density part (darker). This marked difference in density is caused by the wood structure; the latewood is denser than the early wood, which is 

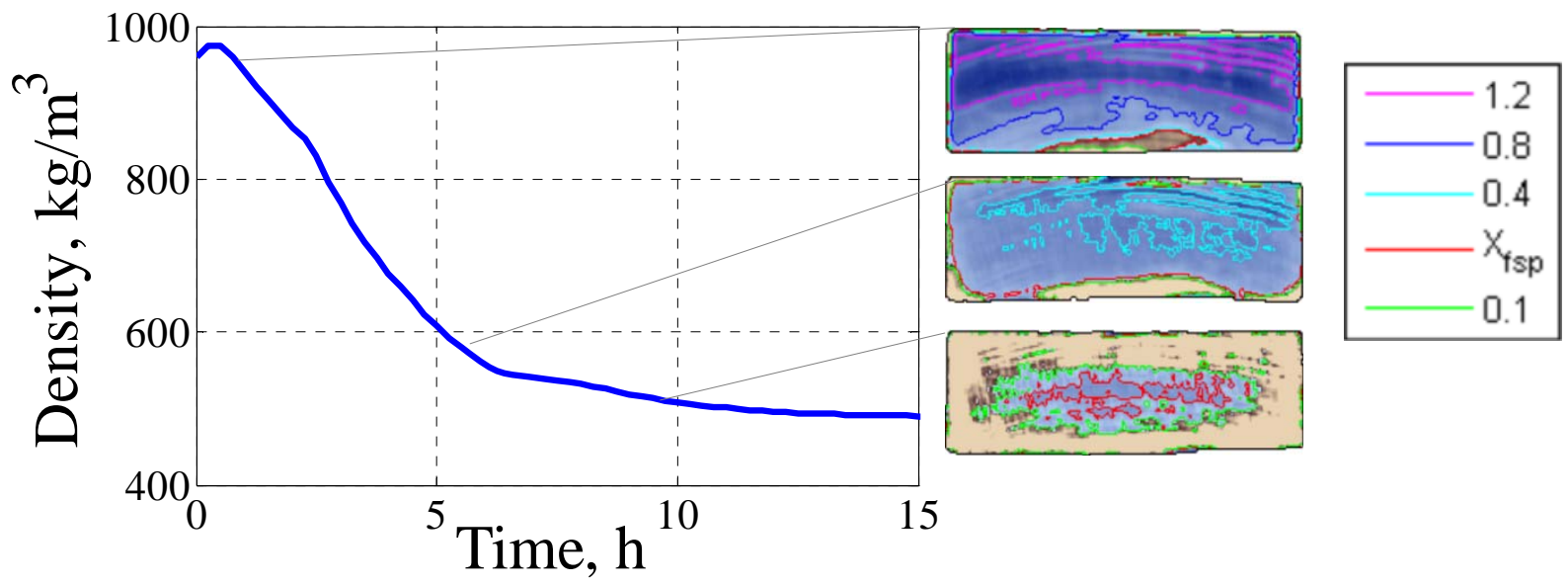

Fig. 6 Density vs. time and dry moisture content profiles.
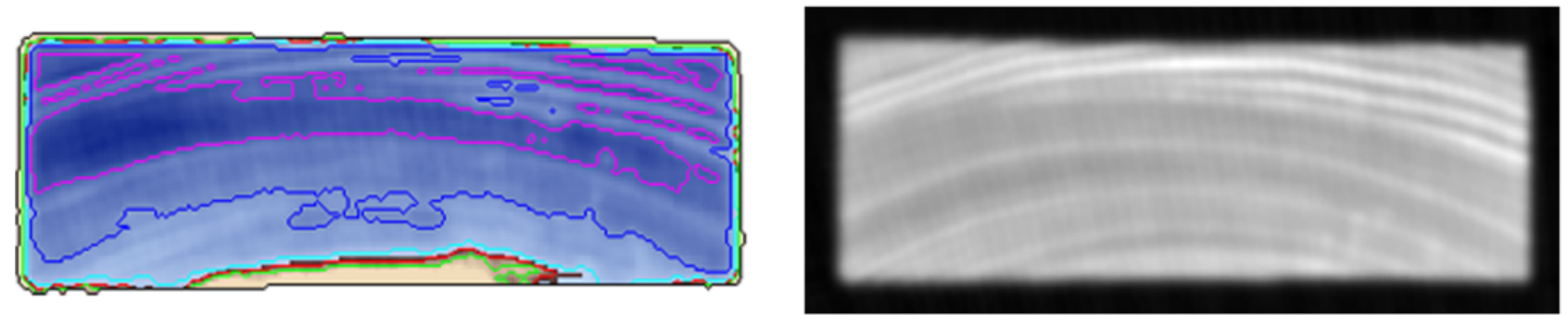

Fig. 7 Moisture content images of a cross-section, after 1.5 hours (left), dry density images of cross section (right).

formed early in the season. Latewood is very thick-walled and has very small cell cavities compared to early wood, which has thin walls and large cell cavities. Thus the early wood could contain a high amount of water, which can be seen in the cross-section's image for the mc (Fig. 7).

\section{Conclusion}

The use of the advanced image-processing algorithm for the moisture content calculation of density data from the CT scanning during high temperature drying in the climate chamber showed that this method is a powerful tool for analyzing the moisture flow within the wood piece. The new CT-scanner together with the climate chamber gave unique results, as it has not been possible to study high temperature drying before. This means that the drying process could be further developed and improved in order to avoid defects as cracks and mc gradients for higher quality on the product.

\section{Acknowledgement}

The authors are grateful to Ångpanneföreningen's Foundation for Research and Development, for funding this project.

\section{References}

[1] L. F. Hawley, Wood-liquid relations, US Department of Agriculture Technical Bulletin, Washington, DC, 1931, p. 248.

[2] S. Pang, Relative importance of vapour diffusion and convective flow in modeling of softwood drying, Drying Technology16 (1) (1998) 271-281.

[3] M. Nabhani, A. Laghdir and Y. Fortin, Simulation of high-temperature drying of wood, Drying Technology 28 (2010) 1142-1147.

[4] J. J. Nijdam, A high-temperature drying model for softwood timber, Chemical Engineering Science 55 (2000) 3585-3598.

[5] P. Wilberg, X-ray CT-scanning of wood during drying, Ph.D. thesis, Division of Wood Physics, Luleå University of Technology, Skellefteå, Sweden. Thesis No. 2001:10.

[6] Medindia Health Network Pvt Ltd. Computed Tomography Basic Working Principle of CT Scan, available online at: http://www.medindia.net/patients/ 
patientinfo/CT_Scan_working.htm\#ixzz1HJiawR00, updated May 18, 2011.

[7] A. B. Valutec, available online at: http://www.valutec.se/, updated 2010.

[8] L. Hansson, Microwave treatment of wood, Ph.D. thesis, Division of Wood Physics, Luleå University of Technology, Skellefteå, Sweden. Thesis No. 2007: 40.

[9] I. Arganda-Carreras, ImageJ plugin for bidirectional elastic registration of two images, available online at: http://biocomp.cnb.csic.es/ iarganda/bUnwarpJ/, updated 2011.

[10] Available at: http://rsbweb.nih.gov/ij/index.html, updated 2011.

[11] J. B. Boutelje, Träfakta. Träteknik Centrum, 1989.

[12] J. F. Siau, Transport Processes in Wood, Springer-Verlag: New York, 1984.

[13] J. F. Siau, Wood: Influence of Moisture On Physical Properties, Virginia Polytechnic Inst. and State Univ.: Blacksburg, VA, 1995. 